\title{
PHYSIOLOGICAL RESPONSE OF SEEDS OF PEANUT GENOTYPES TO WATER DEFICIENCY
}

\author{
RESPOSTA FISIOLÓGICA DE SEMENTES DE GENÓTIPOS DE AMENDOIM \\ SUBMETIDOS A DEFICIÊNCIA HÍDRICA
}

\section{Willians César CARREGA ${ }^{1}$; Pedro de Figueiredo Rocha Barbosa Martins ${ }^{1}$; Allan Lopes Bacha ${ }^{1}$; Anne Elise Cesarin ${ }^{1}$; Thiago Souza Oliveira ${ }^{1}$; Ignácio José de Godoy²; Pedro Luis da Costa Aguiar Alves ${ }^{1}$}

1. Department of Biology Applied to Agriculture, São Paulo State University, UNESP/FCAV, Jaboticabal, SP, Brazil. willianscesar@hotmail.com; 2. Grain and Fiber Center, Agronomic Institute of Campinas, Campinas, SP, Brazil

\begin{abstract}
Among the factors that may influence the germination process of seeds, water deficiency is considered the most important. The present study aims to evaluate the physiological response of peanut genotypes to water deficiency. The experiment was conducted in a completely randomized design with four replications in a $12 \times 7$ factorial scheme with 12 peanut genotypes and seven polyethylene glycol 6000 concentrations corresponding to osmotic potentials of $0.00 ;-0.10 ;-0.20 ;-0.40 ;-0.60 ;-0.80$ and $-1.00 \mathrm{MPa}$. The data were submitted to analysis of variance and Tukey's test at a 5\% probability level and, when significant, to analysis of variance adjusted to a quadratic polynomial curve. The germination percentage, seed vigor evaluated by the speed germination index, mean germination time, root length and root dry matter of some genotypes were affected by the osmotic potentials. Among the genotypes, there is a differential response related to the water deficiency level. In relation to the physiological response of the seeds, the lineages 506, 599, and 870 and the cultivar IAC Tatu-ST have a higher tolerance of water deficit. The peanut genotypes begin to suffer the effects of water deficiency at the osmotic potential of $-0.4 \mathrm{MPa}$.
\end{abstract}

KEYWORDS: Arachis hypogaea L. PEG 6000. Osmotic potential. Water restriction. Germination.

\section{INTRODUCTION}

Among the external factors that may affect seed quality, water deficiency is considered one of the most important, especially during the germination process, because according to Steinbrecher and Leubner-Metzger, (2018), water is responsible for activating metabolic processes involved in all the steps of germination, including primary root protrusion.

Currently, information about the degree of tolerance of peanut seeds to water deficit is scarce. Therefore, it is necessary to perform studies to verify and confirm the performance of genotypes under water deficit conditions. To simulate the germination capacity of seeds under this stressful condition, solutions with different osmotic potentials are used (ZHANG et al., 2018; MORADI et al., 2014; WANG et al., 2012).

Studies demonstrate that seeds under water deficit conditions have a lower germination rate, vigor, and velocity and a higher mean germination time (PELEGRINI et al., 2013; ROSA et al., 2005; BEWLEY; BLACK 1994). Vegetable species exhibit different behaviors in response to water deficit, from being very sensitive to being more resistant (KRATZ et al., 2013). The peanut is an oleaginous plant known for showing a high tolerance of water deficiency that is mainly due to its ability to live in environments with low water availability (PEREIRA et al., 2012; SANTOS et al., 2013). However, this tolerance varies depending on morphological and physiological characteristics (PEREIRA et al., 2016; ARRUDA et al., 2015; SANTOS et al., 2013).

A too-negative osmotic potential, especially at the start of imbibition, alters the water absorption of seeds and may prevent the sequence of events that reactivate metabolic processes during the germination process (BANSAL et al., 1980); this inactivation may in turn harden the seeds and delay or even inhibit the start of germination if the osmotic potential is too negative.

Polyethylene glycol 6000 (PEG 6000) is the most-used product to simulate drought stress, because it is chemically inert and nontoxic to seeds and because its high molecular weight prevents it from being absorbed by seeds (VILLELA et al., 1991).

Germination studies under conditions of water deficiency are of fundamental importance, because they permit the response of seed 
germination to adverse environments to be evaluated and, therefore, may be of help in the decision-making process about the best genotypes to use in agriculture. Given these facts, the present work aimed to evaluate the physiological response of peanut seeds to water deficiency, with the goal of seeking cultivars to be sown in regions that are more affected by drought.

\section{MATERIAL AND METHODS}

The experiment was performed in the Plant Physiology Laboratory of the Departamento de Biologia Aplicada à Agropecuária, São Paulo State University, Campus of Jaboticabal, São Paulo State.

The experiment followed a completely randomized design with eight replications and a 12 X 7 factorial scheme with 12 genotypes (cultivars IAC 503, IAC 505, IAC Runner, IAC 886, IAC OL3, IAC OL4, and IAC Tatu-ST and lines 322, 506, 573, 599, 870 and 967) submitted to seven concentrations of polyethylene glycol 6000 (PEG $6000)$ corresponding to osmotic potentials of 0.00 ; $0.10 ;-0.20 ;-0.40 ;-0.60 ;-0.80$ and $-1.00 \mathrm{MPa}$. For the zero-concentration treatment (control), distilled water was used.

The genotypes (cultivars and lines) used in the study were provided by the Instituto Agronômico de Campinas (IAC), and the osmotic potentials were attained by the concentration levels of PEG 6000 presented in Villela et al. (1991). The tests were performed using eight replications of 25 seeds per treatment. The seeds were previously treated with thiram fungicide (Vitavax®-Thiram $200 \mathrm{SC}, 250 \mathrm{~mL}$ of c.p. $100 \mathrm{~kg}^{-1}$ ). After fungicide treatment, two sheets of filter paper were moistened with a volume of distilled water (control) or PEG 6000 proportional to 2.5 times the weight of dry paper. Seeds were kept in lidded transparent plastic boxes (previously sterilized) of $11.0 \mathrm{~cm} \mathrm{x} 11.0 \mathrm{~cm} \mathrm{x}$ $3.0 \mathrm{~cm}$, which were then closed, sealed with Parafilm® (BrAND, Germany) to reduce humidity loss and kept in a germination chamber at $25^{\circ} \mathrm{C}$.

The germination percentage $(\mathrm{G})$ of seeds was evaluated by defining germinated seeds as those with $2 \mathrm{~mm}$ of radicle protrusion. We calculated the germination percentage, speed index (MAGUIRE, 1962) and mean germination time (MGT; LABOURIAU, 1983). After 15 days, the length of roots was measured, and the dry matter was determined after drying in an air-circulation oven at $70^{\circ} \mathrm{C}$ to constant mass.

The results obtained were submitted to analysis of variance, and the means were compared by Tukey's test at a $5 \%$ probability. When significant differences were detected, means were submitted to regression analysis through OriginPro 8 software. Based on the evaluated characters, a group analysis was performed by a hierarchical method, using Euclidian distance.

\section{RESULTS AND DISCUSSION}

Based the results of germination percentage (G), speed germination index (SGI), mean germination time (MGT), root length and root dry matter, the peanut genotypes behaved differently in response to the water deficiency simulated with PEG 6000. The regression analysis verified that the germination process of all genotypes follows a quadratic polynomial curve (Figures 1, 2 and 3).

For most of the genotypes evaluated, the $\mathrm{G}$ and SGI were affected by osmotic potentials of -0.8 $\mathrm{MPa}$ to -1.0 MPa, except for Runner IAC 886 (-0.4 $\mathrm{MPa}$ to -1.0 $\mathrm{MPa})$, line 870 (-0.6 $\mathrm{MPa}$ to $-1.0 \mathrm{MPa})$ and line 599 (-1.0 MPa) (Figures 1 and 2). However, the genotypes that are negatively affected by the two lowest osmotic potentials (-0.8 MPa and -1.0 MPa) show a better capacity to tolerate environments in which water deficit may occur; thus, these genotypes are considered promising and should be tested for their ability to adapt to environments with water restrictions during plant development. Based on MGT, the germination interval was from 6 to 9 days. Genotypes 506, 599 and OL4 were not affected by the osmotic potentials. For the remaining genotypes, osmotic potentials of -0.8 $\mathrm{MPa}$ and $-1.0 \mathrm{MPa}$ prolonged the interval for germination, except for IAC Tatu-ST, which even in the highest PEG 6000 concentration germinated in approximately 7 days (Figure 3 ).

Among the genotypes, cultivar IAC Tatu-ST and lines 506 and 599 had the highest G and SGI at the highest osmotic potentials $(-0.6 \mathrm{MPa},-0.8 \mathrm{MPa}$ and $-1.0 \mathrm{MPa}$ ) and thus had higher tolerance of water deficient conditions compared to the other genotypes. Based on MGT, these genotypes show lower germination times (Tables 1, 2 and 3), which indicates that they need less water to reactivate their metabolism for germination and thus could be used to establish crops faster. In addition, this lower amount of time reduces the exposure of the seed to insect and pathogen attack during this developmental phase. 

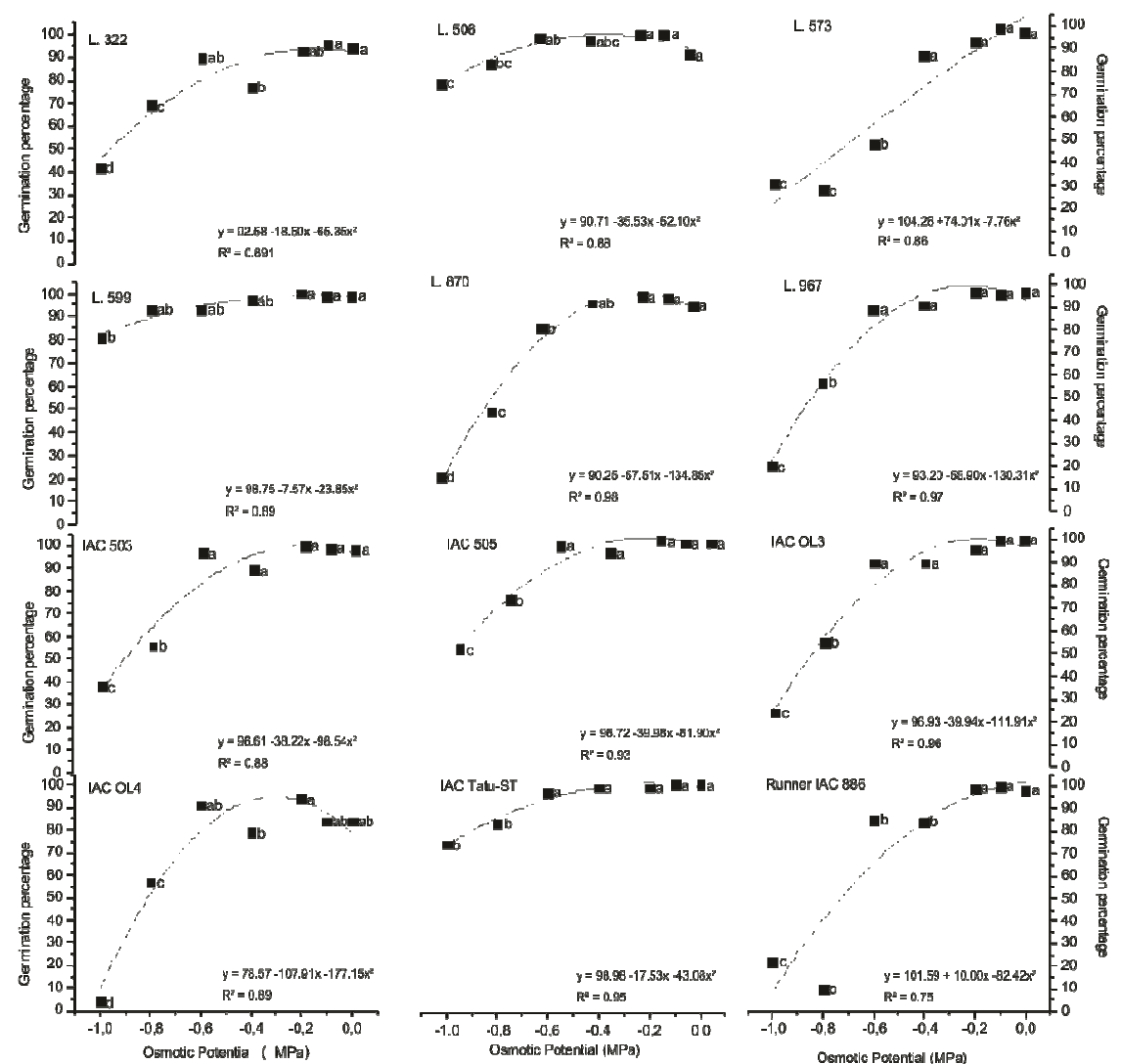

Figure 1. Germination percentage $(\mathrm{G})$ of seeds of peanut genotypes submitted to water deficiency levels simulated with polyethylene glycol 6000. Lowercase letters in each graph represent results of Tukey's test at a $5 \%$ probability.
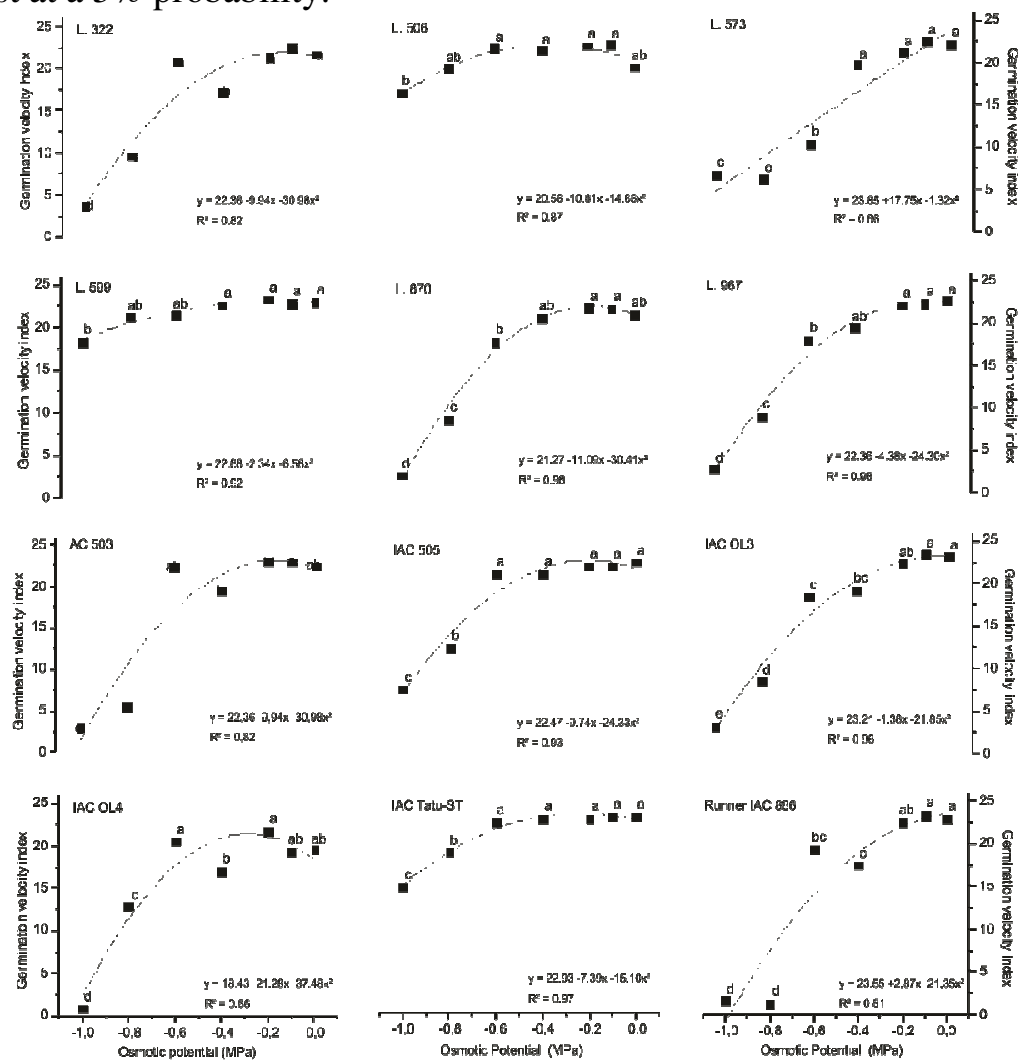

Figure 2. Speed germination index (SGI) of seeds of peanut genotypes submitted to water deficit levels simulated with polyethylene glycol 6000. Lowercase letters in each graph represent results of Tukey's test at a 5\% probability. 


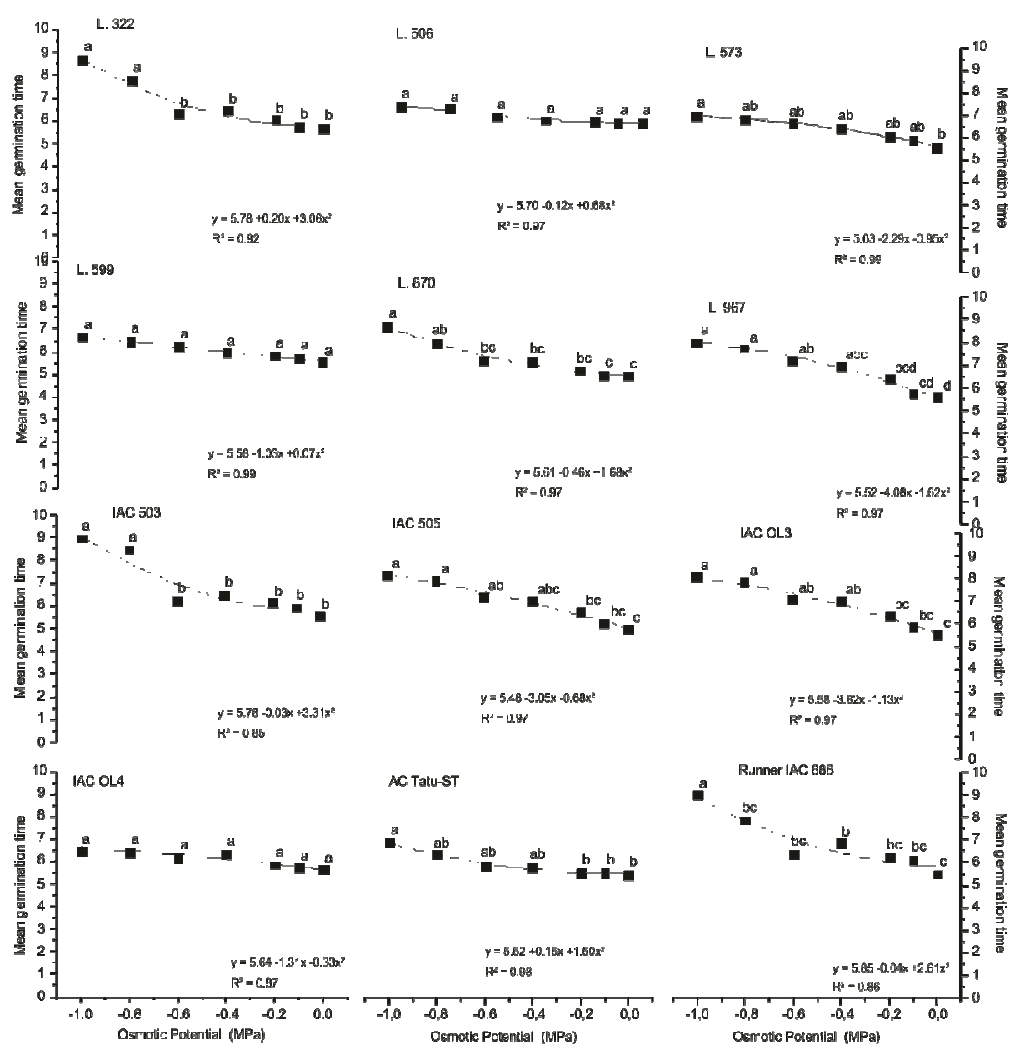

Figure 3. Mean germination time (MGT) of seeds of peanut genotypes submitted to water deficit levels simulated with polyethylene glycol 6000. Lowercase letters in each graph represent results of Tukey's test at $5 \%$ probability.

Table 1. Germination percentage $(\mathrm{G})$ of seeds of peanut genotypes submitted to different osmotic potentials.

\begin{tabular}{llllllll}
\hline \hline \multirow{2}{*}{ Genotype } & \multicolumn{7}{l}{ Osmotic Potential (MPa) } \\
\cline { 2 - 7 } & 0.0 & -0.1 & -0.2 & -0.4 & -0.6 & -0.8 & -1.0 \\
\hline cv. IAC 503 & $98.0 \mathrm{a}$ & $99.0 \mathrm{a}$ & $100.0 \mathrm{a}$ & $89.0 \mathrm{abc}$ & $97.0 \mathrm{a}$ & $56.0 \mathrm{~cd}$ & $38.0 \mathrm{bcd}$ \\
cv. IAC 505 & $99.0 \mathrm{a}$ & $99.0 \mathrm{a}$ & $100.0 \mathrm{a}$ & $95.0 \mathrm{ab}$ & $98.0 \mathrm{a}$ & $75.0 \mathrm{~b}$ & $54.0 \mathrm{~b}$ \\
cv. IAC 886 & $98.0 \mathrm{a}$ & $100.0 \mathrm{a}$ & $99.0 \mathrm{a}$ & $84.0 \mathrm{abc}$ & $85.0 \mathrm{a}$ & $22.0 \mathrm{e}$ & $10.0 \mathrm{f}$ \\
cv. IAC OL3 & $100.0 \mathrm{a}$ & $100.0 \mathrm{a}$ & $96.0 \mathrm{a}$ & $90.0 \mathrm{abc}$ & $90.0 \mathrm{a}$ & $55.0 \mathrm{~cd}$ & $24.0 \mathrm{de}$ \\
cv. IAC OL4 & $84.0 \mathrm{a}$ & $84.0 \mathrm{a}$ & $94.0 \mathrm{a}$ & $79.0 \mathrm{bc}$ & $91.0 \mathrm{a}$ & $57.0 \mathrm{~cd}$ & $4.0 \mathrm{f}$ \\
cv. IAC Tatu & $100.0 \mathrm{a}$ & $100.0 \mathrm{a}$ & $99.0 \mathrm{a}$ & $99.0 \mathrm{a}$ & $97.0 \mathrm{a}$ & $83.0 \mathrm{ab}$ & $74.0 \mathrm{a}$ \\
L. 322 & $94.0 \mathrm{a}$ & $96.0 \mathrm{a}$ & $93.0 \mathrm{a}$ & $77.0 \mathrm{c}$ & $90.0 \mathrm{a}$ & $69.0 \mathrm{bc}$ & $42.0 \mathrm{bc}$ \\
L. 506 & $88.0 \mathrm{a}$ & $97.0 \mathrm{a}$ & $97.0 \mathrm{a}$ & $94.0 \mathrm{abc}$ & $97.0 \mathrm{a}$ & $84.0 \mathrm{ab}$ & $75.0 \mathrm{a}$ \\
L. 573 & $97.0 \mathrm{a}$ & $99.0 \mathrm{a}$ & $93.0 \mathrm{a}$ & $87.0 \mathrm{abc}$ & $48.0 \mathrm{~b}$ & $31.0 \mathrm{de}$ & $28.0 \mathrm{~d}$ \\
L. 599 & $99.0 \mathrm{a}$ & $99.0 \mathrm{a}$ & $100.0 \mathrm{a}$ & $97.0 \mathrm{a}$ & $93.0 \mathrm{a}$ & $93.0 \mathrm{a}$ & $81.0 \mathrm{a}$ \\
L. 870 & $91.0 \mathrm{a}$ & $94.0 \mathrm{a}$ & $95.0 \mathrm{a}$ & $92.0 \mathrm{abc}$ & $81.0 \mathrm{a}$ & $44.0 \mathrm{de}$ & $15.0 \mathrm{ef}$ \\
L. 967 & $97.0 \mathrm{a}$ & $96.0 \mathrm{a}$ & $97.0 \mathrm{a}$ & $91.0 \mathrm{abc}$ & $89.0 \mathrm{a}$ & $57.0 \mathrm{~cd}$ & $20.0 \mathrm{ef}$ \\
\hline F & $1.81^{\mathrm{ns}}$ & $1.38^{\mathrm{ns}}$ & $0.50^{\mathrm{ns}}$ & $3.15^{* *}$ & $12.43^{* *}$ & $39.12^{* *}$ & $44.78^{* *}$ \\
\hline \hline
\end{tabular}

Means followed by the same letter in a column do not differ at a $5 \%$ probability, based on Tukey's test. From an $\mathrm{F}$ test, **significant at $1 \%$ probability; *significant at 5\% probability; ${ }^{\text {ns }}$ nonsignificant; cv. (cultivar); L. (line); CV (\%): 9.49; MDS: 6.77.

Table 2. Speed germination index (SGI) of seeds of peanut genotypes submitted to different osmotic potentials.

\begin{tabular}{llllllll}
\hline \hline \multirow{2}{*}{ Genotype } & \multicolumn{7}{l}{ Osmotic Potential (MPa) } \\
\cline { 2 - 8 } & 0.0 & -0.1 & -0.2 & -0.4 & -0.6 & -0.8 & -1.0 \\
\hline cv. IAC 503 & $22.5 \mathrm{ab}$ & $22.9 \mathrm{ab}$ & $23.0 \mathrm{a}$ & $19.3 \mathrm{abcd}$ & $22.7 \mathrm{a}$ & $5.5 \mathrm{~d}$ & $2.9 \mathrm{~d}$ \\
cv. IAC 505 & $23.2 \mathrm{ab}$ & $22.8 \mathrm{ab}$ & $22.8 \mathrm{a}$ & $21.9 \mathrm{ab}$ & $21.9 \mathrm{ab}$ & $13.1 \mathrm{~b}$ & $8.2 \mathrm{~b}$ \\
cv. IAC 886 & $23.0 \mathrm{ab}$ & $23.4 \mathrm{a}$ & $22.8 \mathrm{a}$ & $17.5 \mathrm{~cd}$ & $19.4 \mathrm{abc}$ & $1.2 \mathrm{e}$ & $1.4 \mathrm{~d}$ \\
cv. IAC OL3 & $23.2 \mathrm{a}$ & $23.4 \mathrm{a}$ & $22.3 \mathrm{a}$ & $19.3 \mathrm{bcd}$ & $18.5 \mathrm{ab}$ & $8.5 \mathrm{~cd}$ & $3.3 \mathrm{~cd}$ \\
cv. IAC OL4 & $19.6 \mathrm{~b}$ & $19.4 \mathrm{~b}$ & $21.7 \mathrm{a}$ & $17.1 \mathrm{~d}$ & $20.6 \mathrm{abc}$ & $12.9 \mathrm{~b}$ & $0.1 \mathrm{~d}$
\end{tabular}




\begin{tabular}{lccccccc}
\hline \hline cv. IAC Tatu & $23.4 \mathrm{a}$ & $23.4 \mathrm{a}$ & $23.1 \mathrm{a}$ & $23.2 \mathrm{a}$ & $22.7 \mathrm{a}$ & $19.2 \mathrm{a}$ & $15.0 \mathrm{a}$ \\
L. 322 & $21.7 \mathrm{ab}$ & $22.5 \mathrm{ab}$ & $21.3 \mathrm{a}$ & $17.3 \mathrm{~cd}$ & $20.8 \mathrm{abc}$ & $9.6 \mathrm{bc}$ & $3.8 \mathrm{~cd}$ \\
L. 506 & $19.8 \mathrm{ab}$ & $22.5 \mathrm{ab}$ & $22.4 \mathrm{a}$ & $21.8 \mathrm{ab}$ & $22.1 \mathrm{ab}$ & $19.7 \mathrm{a}$ & $16.8 \mathrm{a}$ \\
L. 573 & $22.2 \mathrm{ab}$ & $22.5 \mathrm{ab}$ & $21.3 \mathrm{a}$ & $19.7 \mathrm{abcd}$ & $10.4 \mathrm{~d}$ & $6.2 \mathrm{~cd}$ & $6.7 \mathrm{bc}$ \\
L. 599 & $23.0 \mathrm{ab}$ & $22.8 \mathrm{ab}$ & $23.3 \mathrm{a}$ & $22.7 \mathrm{ab}$ & $21.5 \mathrm{abc}$ & $21.3 \mathrm{a}$ & $18.4 \mathrm{a}$ \\
L. 870 & $21.3 \mathrm{ab}$ & $22.0 \mathrm{ab}$ & $22.1 \mathrm{a}$ & $20.9 \mathrm{abc}$ & $18.2 \mathrm{c}$ & $9.1 \mathrm{~cd}$ & $2.6 \mathrm{~d}$ \\
L. 967 & $22.7 \mathrm{ab}$ & $22.3 \mathrm{ab}$ & $22.2 \mathrm{a}$ & $19.5 \mathrm{bcd}$ & $18.0 \mathrm{c}$ & $9.0 \mathrm{~cd}$ & $2.8 \mathrm{~d}$ \\
\hline F & $2.77^{* *}$ & $1.87^{*}$ & $0.75^{\text {ns }}$ & $7.21^{* *}$ & $18.64^{* *}$ & $62.17^{* *}$ & $65.16^{* *}$ \\
\hline \hline
\end{tabular}

Means followed by the same letter in a column do not differ at a 5\% probability, based on Tukey's test. From an $\mathrm{F}$ test, **significant at $1 \%$ probability; *significant at 5\% probability; ${ }^{\text {ns }}$ nonsignificant; cv. (cultivar); L. (line); CV (\%): 8.75; MDS: 1.38.

Table 3. Mean germination time (MGT) of seeds of peanut genotypes submitted to different osmotic potentials.

\begin{tabular}{lccccccc}
\hline \multirow{2}{*}{ Genotype } & \multicolumn{7}{l}{ Osmotic Potential (MPa) } \\
\cline { 2 - 7 } & 0.0 & -0.1 & -0.2 & -0.4 & -0.6 & -0.8 & -1.0 \\
\hline cv. IAC 503 & $5.56 \mathrm{a}$ & $5.93 \mathrm{a}$ & $6.15 \mathrm{a}$ & $6.49 \mathrm{a}$ & $6.24 \mathrm{a}$ & $8.48 \mathrm{a}$ & $8.99 \mathrm{a}$ \\
cv. IAC 505 & $5.45 \mathrm{a}$ & $5.73 \mathrm{a}$ & $6.18 \mathrm{a}$ & $6.68 \mathrm{a}$ & $6.86 \mathrm{a}$ & $7.58 \mathrm{abc}$ & $7.86 \mathrm{abc}$ \\
cv. IAC 886 & $5.50 \mathrm{a}$ & $6.13 \mathrm{a}$ & $6.25 \mathrm{a}$ & $6.83 \mathrm{a}$ & $6.37 \mathrm{a}$ & $6.93 \mathrm{bcd}$ & $9.00 \mathrm{a}$ \\
cv. IAC OL3 & $5.56 \mathrm{a}$ & $5.85 \mathrm{a}$ & $6.34 \mathrm{a}$ & $7.02 \mathrm{a}$ & $7.09 \mathrm{a}$ & $7.84 \mathrm{ab}$ & $8.08 \mathrm{ab}$ \\
cv. IAC OL4 & $5.65 \mathrm{a}$ & $5.73 \mathrm{a}$ & $5.86 \mathrm{a}$ & $6.33 \mathrm{a}$ & $6.17 \mathrm{a}$ & $6.43 \mathrm{~cd}$ & $6.48 \mathrm{c}$ \\
cv. IAC Tatu & $5.47 \mathrm{a}$ & $5.56 \mathrm{a}$ & $5.55 \mathrm{a}$ & $5.80 \mathrm{a}$ & $5.88 \mathrm{~b}$ & $6.34 \mathrm{~d}$ & $6.90 \mathrm{bc}$ \\
L. 322 & $5.66 \mathrm{a}$ & $5.77 \mathrm{a}$ & $6.05 \mathrm{a}$ & $6.45 \mathrm{a}$ & $6.29 \mathrm{a}$ & $7.81 \mathrm{ab}$ & $8.69 \mathrm{a}$ \\
L. 506 & $5.73 \mathrm{a}$ & $5.70 \mathrm{a}$ & $5.76 \mathrm{a}$ & $5.83 \mathrm{a}$ & $5.99 \mathrm{ab}$ & $6.33 \mathrm{~d}$ & $6.46 \mathrm{c}$ \\
L. 573 & $5.57 \mathrm{a}$ & $5.90 \mathrm{a}$ & $6.06 \mathrm{a}$ & $6.41 \mathrm{a}$ & $6.64 \mathrm{a}$ & $6.81 \mathrm{bcd}$ & $7.00 \mathrm{bc}$ \\
L. 599 & $5.54 \mathrm{a}$ & $5.72 \mathrm{a}$ & $5.83 \mathrm{a}$ & $5.94 \mathrm{a}$ & $6.21 \mathrm{a}$ & $6.48 \mathrm{bcd}$ & $6.66 \mathrm{bc}$ \\
L. 870 & $5.57 \mathrm{a}$ & $5.62 \mathrm{a}$ & $5.83 \mathrm{a}$ & $6.22 \mathrm{a}$ & $6.30 \mathrm{a}$ & $7.03 \mathrm{abcd}$ & $7.76 \mathrm{abc}$ \\
L. 967 & $5.56 \mathrm{a}$ & $5.72 \mathrm{a}$ & $6.43 \mathrm{a}$ & $7.00 \mathrm{a}$ & $7.24 \mathrm{a}$ & $7.76 \mathrm{abc}$ & $8.00 \mathrm{ab}$ \\
\hline F & $0.06^{\mathrm{ns}}$ & $0.24^{\mathrm{ns}}$ & $0.70^{\text {ns }}$ & $1.82^{\mathrm{ns}}$ & $1.88^{*}$ & $6.58^{* *}$ & $24.38^{* *}$ \\
\hline \hline
\end{tabular}

Means followed by the same letter in a column do not differ at a $5 \%$ probability, based on Tukey's test. From an $\mathrm{F}$ test, **significant at $1 \%$ probability; *significant at 5\% probability; ${ }^{\text {ns }}$ nonsignificant; cv. (cultivar); L. (line); CV (\%): 9.80; MDS: 0.55.

Based on the root length of peanut seedlings (Table 4), osmotic potentials of -0.6 MPa to -1.0 $\mathrm{MPa}$ greatly reduce root development compared to the other potentials. Among the genotypes, line 870 performed better when submitted to osmotic potentials of $-0.4,-0.6$, and $-0.8 \mathrm{MPa}$. When the seedlings were submitted to the highest osmotic potential (-1.0 MPa), the most tolerant genotype was IAC Tatu-ST.

Osmotic potentials of $-0.6 \mathrm{MPa}$ to $-1.0 \mathrm{MPa}$ reduced root dry matter the most. Among all osmotic potentials $(0.0$ to $-1.0 \mathrm{MPa})$, there was no significant difference for line 599, indicating that this genotype has a higher tolerance of water deficit. At the highest potentials (-0.4 MPa to $-1.0 \mathrm{MPa}$ ), there was no significant difference among the genotypes evaluated (Table 5).

The dendrogram resulting from the cluster analysis indicates, based on the evaluated characters G, SGI, MGT, root length and root dry matter, the genotypes submitted to osmotic potentials formed four groups. The first group (G1) was formed by genotypes that performed better at the water deficit levels. In this group, most of the genotypes performed best at the lowest osmotic potentials; however, there is a subgroup (with better performance at higher osmotic potentials) that shows a similarity to the less-stressed genotypes, and this subgroup is therefore highlighted for showing better tolerance than the other evaluated genotypes. In this subgroup, genotypes 506, 509, Tatu-ST and 870 are highlighted. The second group (G2) was formed by the genotypes (506 and 573) that performed best when submitted to the lowest osmotic potentials. The third group (G3) was formed by genotypes with higher susceptibility to higher levels of water deficiency, including all genotypes susceptible to higher osmotic potentials, except for 506 and 599, which are not susceptible. The fourth group (G4) was formed only by the IAC OL4 cultivar, which showed the highest susceptibility among all genotypes (Figure 4). 
Table 4. Interaction analysis of effects of the main factors, genotypes and osmotic potentials, on the root length (mm) of peanut seedlings.

\begin{tabular}{|c|c|c|c|c|c|c|c|c|c|c|c|c|c|c|c|}
\hline \multirow{2}{*}{ Genotype } & \multicolumn{15}{|c|}{ Osmotic Potential (MPa) } \\
\hline & 0.0 & -0.1 & & -0.2 & & -0.4 & & -0.6 & & -0.8 & & -1.0 & & $\mathrm{~F}$ & \\
\hline cv. IAC 503 & 39.7 & $\mathrm{CD}$ a & 38.8 & $\mathrm{BC} \mathrm{a}$ & 41.5 & $\mathrm{AB}$ a & 39.5 & $\mathrm{~B} \mathrm{a}$ & 14.7 & $\mathrm{EF} b$ & 7.5 & $\mathrm{D} \mathrm{b}$ & 5.6 & $\mathrm{~B} \mathrm{~b}$ & $51.97 * *$ \\
\hline cv. IAC 505 & 76.2 & $\mathrm{~A} \mathrm{a}$ & 53.6 & $\mathrm{Ab}$ & 45.1 & $\mathrm{AB} b$ & 33.4 & $\mathrm{BC} \mathrm{c}$ & 35.1 & $\mathrm{AB} \mathrm{c}$ & 14.5 & $\mathrm{BCD} d$ & 6.9 & $\mathrm{AB} d$ & $44.27 * *$ \\
\hline cv. IAC 886 & 43.8 & $\mathrm{CD}$ a & 44.3 & $\mathrm{AB}$ a & 46.9 & $\mathrm{~A} \mathrm{a}$ & 42.2 & $\mathrm{AB}$ a & 26.6 & $\mathrm{BC} b$ & 8.5 & D c & 5.4 & $\mathrm{~B} \mathrm{c}$ & $59.59 * *$ \\
\hline cv. IAC OL3 & 37.2 & $\mathrm{CD}$ a & 24.9 & $\mathrm{Db}$ & 25.3 & $\mathrm{DE} b$ & 27.3 & $\mathrm{CD} \mathrm{b}$ & 24.0 & $\mathrm{CDE} b$ & 10.9 & $\mathrm{CD} \mathrm{c}$ & 7.7 & $\mathrm{AB} \mathrm{c}$ & $40.78 * *$ \\
\hline cv. IAC OL4 & 38.0 & $\mathrm{CD}$ a & 35.0 & $\mathrm{BCD} a b$ & 30.1 & CDE abc & 32.7 & $\mathrm{BC}$ abc & 28.0 & $\mathrm{ABC} b c$ & 24.6 & $\mathrm{AB} \mathrm{c}$ & 5.4 & B d & $21.84 * *$ \\
\hline cv. IAC Tatu & 37.6 & $\mathrm{CD}$ a & 36.5 & $\mathrm{BC}$ ab & 34.2 & $\mathrm{BCD} a b$ & 32.5 & $\mathrm{BC} a b$ & 27.0 & $\mathrm{BC} b \mathrm{c}$ & 21.0 & $\mathrm{ABC} c \mathrm{~d}$ & 16.6 & $\mathrm{Ad}$ & $12.39 * *$ \\
\hline L. 322 & 43.7 & $\mathrm{CD}$ a & 44.0 & $\mathrm{AB}$ a & 40.3 & $\mathrm{ABC} \mathrm{a}$ & 23.9 & $\mathrm{CD} \mathrm{b}$ & 26.7 & $\mathrm{BC} b$ & 11.7 & $\mathrm{CD} \mathrm{c}$ & 6.3 & $\mathrm{AB} \mathrm{c}$ & $103.76^{* *}$ \\
\hline L. 506 & 24.0 & $\mathrm{E}$ a & 14.0 & $\mathrm{~Eb}$ & 13.2 & $\mathrm{~F} \mathrm{~b}$ & 13.0 & $\mathrm{~Eb}$ & 11.5 & $\mathrm{~F} \mathrm{~b}$ & 10.1 & $\mathrm{D} b$ & 9.8 & $\mathrm{AB} b$ & $19.08^{* *}$ \\
\hline L. 573 & 39.6 & $\mathrm{CD}$ a & 29.9 & $\mathrm{CD}$ ab & 29.0 & $\mathrm{DE} b$ & 20.8 & $\mathrm{DE} b \mathrm{c}$ & 16.1 & DEF cd & 10.5 & $\mathrm{CD} d$ & 15.6 & $\mathrm{AB} c \mathrm{~cd}$ & $91.47 * *$ \\
\hline L. 599 & 33.1 & $\mathrm{DE}$ a & 24.3 & $\mathrm{DE} a b$ & 22.9 & $\mathrm{EF} b$ & 24.2 & $\mathrm{CD} a b$ & 17.4 & CDEF b & 15.9 & $\mathrm{ABCD} b$ & 15.0 & $\mathrm{AB} b$ & $19.52 * *$ \\
\hline L. 870 & 44.0 & $\mathrm{Cab}$ & 43.3 & $\mathrm{AB}$ a & 40.5 & $\mathrm{ABC}$ a & 42.7 & $\mathrm{AB}$ a & 38.0 & $\mathrm{Ab}$ & 26.1 & $\mathrm{Ac}$ & 6.5 & $\mathrm{AB} \mathrm{d}$ & $4.44 * *$ \\
\hline L. 967 & 64.2 & $\mathrm{~B}$ a & 53.1 & $\mathrm{Ab}$ & 26.6 & $\mathrm{DE} \mathrm{c}$ & 24.8 & $\mathrm{CD} \mathrm{c}$ & 21.3 & CDEF c & 7.1 & $\mathrm{Dd}$ & 6.0 & $\mathrm{AB} d$ & $7.57 * *$ \\
\hline $\mathrm{F}$ & $36.38 * *$ & $26.20 * *$ & & $18.60 *$ & & $21.34 * *$ & & 12.3 & & $8.14 * *$ & & $3.49 * *$ & & - & \\
\hline
\end{tabular}

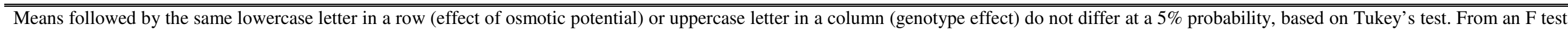

**significant at $1 \%$ probability; *significant at 5\% probability; ${ }^{\mathrm{ns}}$ nonsignificant; cv. (cultivar); L. (line); CV (\%): 17.07; MDS: 4.06. 
Table 5. Interaction analysis of effects of the main factors, genotypes and osmotic potentials, on the dry matter (g) of peanut seedlings.

\begin{tabular}{|c|c|c|c|c|c|c|c|c|c|c|c|c|c|c|c|}
\hline \multirow{2}{*}{ Genotype } & \multicolumn{15}{|c|}{ Osmotic Potential (MPa) } \\
\hline & 0.0 & & -0.1 & & -0.2 & & -0.4 & & $-0,6$ & & $-0,8$ & & $-1,0$ & & $\mathrm{~F}$ \\
\hline cv. IAC 503 & 0.178 & $\mathrm{ABC}$ a & 0.149 & $\mathrm{AB}$ a & 0.130 & $\mathrm{AB} a b$ & 0.103 & $\mathrm{AB} a b c$ & 0.053 & $\mathrm{~A} \mathrm{bc}$ & 0.034 & $\mathrm{Ac}$ & 0.027 & $\mathrm{Ac}$ & $9,41 * *$ \\
\hline cv. IAC 505 & 0.186 & $\mathrm{ABC} \mathrm{a}$ & 0.159 & $\mathrm{~A} a b$ & 0.120 & $\mathrm{AB} a b c$ & 0.101 & $\mathrm{AB}$ bcd & 0.074 & A cde & 0.033 & A de & 0.019 & $\mathrm{~A} \mathrm{e}$ & $10,17 * *$ \\
\hline cv. IAC 886 & 0.209 & $\mathrm{AB}$ a & 0.152 & $\mathrm{~A} a b$ & 0.128 & $\mathrm{AB} a b$ & 0.085 & $\mathrm{AB} b \mathrm{c}$ & 0.043 & $\mathrm{Ac}$ & 0.024 & $\mathrm{Ac}$ & 0.016 & $\mathrm{Ac}$ & $13,88^{* *}$ \\
\hline cv. IAC OL3 & 0.160 & $\mathrm{ABC} \mathrm{a}$ & 0.147 & $\mathrm{AB} a b$ & 0.110 & $\mathrm{AB} a b$ & 0.088 & $\mathrm{AB} a b c$ & 0.076 & $\mathrm{~A} b c$ & 0.024 & $\mathrm{Ac}$ & 0.021 & $\mathrm{Ac}$ & $7,88^{* *}$ \\
\hline cv. IAC OL4 & 0.219 & $\mathrm{~A} \mathrm{a}$ & 0.153 & $\mathrm{~A} a b$ & 0.131 & $\mathrm{AB} b \mathrm{c}$ & 0.109 & $\mathrm{AB}$ bcd & 0.087 & A bcd & 0.054 & A cd & 0.031 & $\mathrm{Ad}$ & $10,67 * *$ \\
\hline cv. IAC Tatu & 0.210 & $\mathrm{AB}$ a & 0.184 & $\mathrm{~A} a b$ & 0.172 & $\mathrm{~A} a b$ & 0.153 & $\mathrm{~A} a b$ & 0.127 & $\mathrm{~A} b c$ & 0.062 & A cd & 0.039 & $\mathrm{Ad}$ & $10,76^{* * *}$ \\
\hline L. 322 & 0.205 & $\mathrm{ABC} a$ & 0.158 & $\mathrm{~A} a b$ & 0.106 & $\mathrm{AB} b \mathrm{c}$ & 0.080 & $\mathrm{AB} b c$ & 0.051 & $\mathrm{Ac}$ & 0.042 & $\mathrm{Ac}$ & 0.031 & $\mathrm{Ac}$ & $11,10^{* * *}$ \\
\hline L. 506 & 0.130 & $\mathrm{ABC} \mathrm{a}$ & 0.100 & $\mathrm{C}$ ab & 0.080 & $\mathrm{~B} a b$ & 0.062 & $\mathrm{~B} a b$ & 0.045 & $\mathrm{Ab}$ & 0.044 & $\mathrm{Ab}$ & 0.033 & $\mathrm{Ab}$ & $3,25 * *$ \\
\hline L. 573 & 0.116 & $\mathrm{Cb}$ & 0.155 & A a & 0.074 & $\mathrm{~B} \mathrm{~b}$ & 0.066 & $\mathrm{AB} b$ & 0.053 & $\mathrm{Ab}$ & 0.045 & $\mathrm{Ab}$ & 0.036 & $\mathrm{Ab}$ & $15,43 * *$ \\
\hline L. 599 & 0.125 & $\mathrm{BC}$ a & 0.104 & $\mathrm{C} \mathrm{a}$ & 0.094 & $\mathrm{AB}$ a & 0.087 & $\mathrm{AB} a$ & 0.075 & $\mathrm{~A} \mathrm{a}$ & 0.067 & $\mathrm{~A} \mathrm{a}$ & 0.052 & $\mathrm{~A} \mathrm{a}$ & $1,56^{\mathrm{ns}}$ \\
\hline L. 870 & 0.181 & $\mathrm{ABC} \mathrm{a}$ & 0.141 & $\mathrm{AB} a b$ & 0.125 & $\mathrm{AB} a b$ & 0.095 & $\mathrm{AB} b c$ & 0.062 & $\mathrm{~A} b c$ & 0.035 & $\mathrm{Ac}$ & 0.017 & $\mathrm{Ac}$ & $9,44 * *$ \\
\hline L. 967 & 0.179 & $\mathrm{ABC} \mathrm{a}$ & 0.154 & $\mathrm{~A} a b$ & 0.086 & $\mathrm{AB} b c$ & 0.068 & $\mathrm{AB} \mathrm{c}$ & 0.054 & $\mathrm{Ac}$ & 0.030 & $\mathrm{Ac}$ & 0.013 & $\mathrm{Ac}$ & $10,15^{* * *}$ \\
\hline $\mathrm{F}$ & $3.27 * *$ & & $4.05 * *$ & & $2.00 *$ & & $1.59^{\mathrm{ns}}$ & & $1.45^{\mathrm{ns}}$ & & $0.52^{\text {ns }}$ & & $035^{\mathrm{ns}}$ & & ----- \\
\hline
\end{tabular}

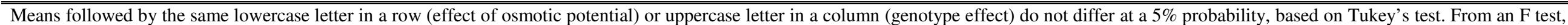

**significant at $1 \%$ probability; *significant at $5 \%$ probability; ${ }^{\text {ns }}$ nonsignificant; cv. (cultivar); L. (line); CV (\%): 30.00; MDS: 0.03 . 


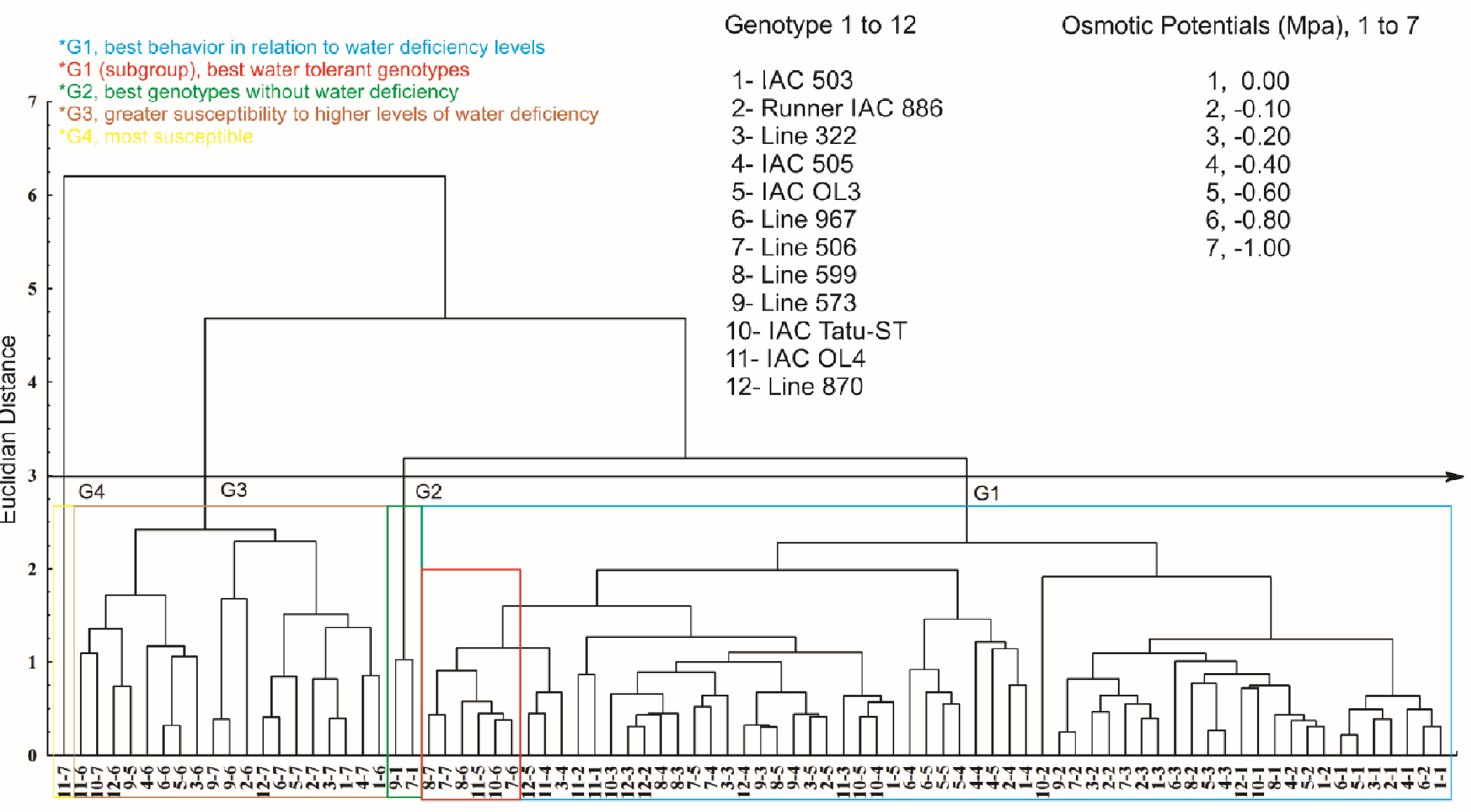

Figure 4. Cluster analysis of groups (G1 to G4) based on the variables germination percentage, first observation of germination, germination velocity index, mean germination time, root length and root dry matter for 12 peanut genotypes submitted to water deficiency (osmotic potentials). 
When seeds are exposed to severe water deficiency caused by too-negative osmotic potentials, especially during the imbibing stage, a reduction in water absorption by the seeds occurs that prevents the reactivation of metabolic processes and in turn has a negative effect on germination. This behavior can be observed in Figure 1, which shows the negative effect of increasing water deficiency on germination.

In this study, it was observed that the germination of peanut seeds submitted to water deficiency simulated with PEG 6000 differed based on genotype. As stated by Kratz et al. (2013), plant species exhibit different behaviors, from being too sensitive to the most resistant to water deficiency. According to Azerêdo et al. (2016), there is a critical water potential that inhibits the germination process for each species.

This study revealed that, even in the same species, different osmotic potentials, especially those that are more negative, affect the germination process of seeds. For the evaluated genotypes, the critical osmotic potential varies from -0.6 $\mathrm{MPa}$ (most susceptible) and -1.0 MPa (most tolerant). These data are similar to those for Erythrina falcata (Fabaceae) obtained by Pelegrini et al. (2013), which verifies that germination potential decreases if osmotic potential reaches $-0.8 \mathrm{MPa}$ (PEG 6000) and is inhibited at $-1.0 \mathrm{MPa}$. In some cases, depending on the species, inhibition may occur even at higher osmotic potentials. Rosa et al. (2005), studying Ateleia glazioviana, which is also in the Fabaceae family, found a delay in the germination process when seeds were submitted to osmotic potentials below $-0.4 \mathrm{MPa}$.

Studies revealed that seeds under water deficit conditions exhibit a reduction in not only germination percentage but also vigor, speed of germination and mean germination time (PELEGRINI et al., 2013; ROSA et al., 2005; BEWLEY; BLACK 1994).

In this study, a lower SGI occurred as water deficit increased (Figure 3). This SGI reduction may be explained by the reduction in seed metabolism due to less available water. Because there is an effect of water reduction on hydric potential, seeds initiate the germination process and, not having enough water to digest their energy reserves and translocate metabolized products (a process known as the triphasic germination pattern) (BEWLEY; BLACK, 1994), the death of the embryo may occur. In addition, during the period in which seeds remain in the soil and do not emerge as a consequence of water deficiency, they are exposed to soil insects and phytopathogens, which may have negative effects on crop establishment.

For MGT, genotypes were similar at concentrations of $0.0,-0.1,-0.2$ and $-0.4 \mathrm{MPa}$. However, as the osmotic potential rises, there is a reduction in water absorption speed by the seeds, which increases the time needed to reach the minimum water level to initiate the germination process (CARVALHO; NAKAGAWA, 2000). This increase in germination time exposes seeds to soil insects and phytopathogens and may compromise crop planting, particularly in sugarcane renewal areas. These results corroborate those observed by Pelegrini et al. (2013) in E. falcata and verify that, at an osmotic potential of at least $-0.8 \mathrm{MPa}$ (PEG 6000), there is a negative effect that leads to a progressive rise in mean germination time.

Growth and root dry matter are two of the adaptive characteristics most often used to select drought-resistant cultivars. Peanut is a leguminous plant with a profound root system and has a great root distribution in the soil profile. According to Aninbon et al. (2016), a larger root system may help increase the ability of a given plant to overcome a drought period. In this study, it was possible to observe differences between genotypes and conclude that all the genotypes experienced reductions in root growth and development when submitted to water deficit. The results obtained corroborate those observed in previous literature that water deficiency diminishes the dry matter produced by peanut genotypes (ARRUDA et al., 2015; KOOLACHART et al., 2013).

\section{CONCLUSIONS}

Regarding the physiological response of seeds, lines 506, 599, and 870 and cultivar IAC Tatu-ST are the most tolerant of water deficiency.

All genotypes except line 573 are tolerant of water deficiency up to the osmotic potential of -0.6 $\mathrm{MPa}$, although they begin to suffer the effects of water deficiency at $-0.4 \mathrm{MPa}$.

\section{ACKNOWLEDGEMENTS}

We thank the Conselho Nacional de Desenvolvimento Científico e Tecnológico (CNPq), which provided scholarships to the first (Proc. n.142462/2013-6) and last (Proc. n.311281/2013-3) authors. 
RESUMO: Entre os fatores externos que podem influenciar o processo germinativo das sementes, a deficiência hídrica é considerada o mais importante. Dessa forma, o objetivo do presente trabalho foi avaliar a resposta fisiológica de sementes de genótipos de amendoim submetidos à deficiência hídrica. $\mathrm{O}$ experimento foi conduzido em esquema fatorial 12 × 7 , tendo como tratamentos sementes de 12 genótipos de amendoim submetidos a sete concentrações de polietilenoglicol 6000 , nos potenciais osmóticos correspondentes a 0,00 ; 0,$10 ;-0,20 ;-0,40 ;-0,60 ;-0,80 ;-1,00 \mathrm{MPa}$. Constatou-se que os genótipos são afetados pelos potenciais osmóticos e, entre os genótipos, há resposta diferenciada em relação ao nível de deficiência hídrica. Em relação à resposta fisiológica das sementes, as linhagens 506, 599, 870 e a cultivar IAC Tatu-ST possuem maior tolerância à deficiência hídrica. Todos os genótipos de amendoim começam a sofrerem os efeitos da deficiência hídrica a partir do potencial osmótico de -0,4 MPa para a maior parte das características avaliadas.

PALAVRAS-CHAVE: Arachis hypogaea L. PEG 6000. Potenciais osmóticos. Restrição hídrica. Germinação.

\section{REFERENCES}

ANINBON, C.; JOGLOY, S.; VORASOOT, N.; PATANOTHAI, A.; NUCHADOMRONG, S.; SENAWONG, $T$. Effect of end of season water deficit on phenolic compounds in peanut genotypes with different levels of resistance to drought. Food chemistry, v. 196, p. 123-129, 2016.

https://doi.org/10.1016/j.foodchem.2015.09.022

ARRUDA, I. M.; CIRINO, V. M.; BURATTO, J. S.; FERREIRA, J. M. Crescimento e produtividade de cultivares e linhagens de amendoim submetidas a déficit hídrico. Pesquisa Agropecuária Tropical, v. 45, n. 2 , p. 146-154, 2015. http:/www.scielo.br/scielo.php?script=sci_arttext\&pid=S198340632015000200009\&lang=pt https://doi.org/10.1590/1983-40632015v4529652

AZERÊDO, G. A.; PAULA, R. C.; VALERI, S. V. Germinação de sementes de Piptadenia moniliformis Benth. sob estresse hídrico. Ciência Florestal, v. 26, n. 1, p. 193-202, 2016.

http://www.redalyc.org/html/534/53444621016/ https://doi.org/10.5902/1980509821112

AZEVEDO NETO, A. D.; NOGUEIRA, R. J. M. C.; MELO FILHO, P. A.; SANTOS, R. C. Physiological and biochemical responses of peanut genotypes to water deficit. Journal of Plant Interactions, v. 5, n. 1, p. 1-10, 2010. http://www.tandfonline.com/doi/abs/10.1080/17429140902999243

BANSAL, R. P.; BHATI, P. R.; SEN, D. N. Differential specificity in water inhibition of Indian arid zone. Biologia Plantarum, v. 22, n. 5, p. 327-331, 1980. https://link.springer.com/article/10.1007/BF02908976

BEWLEY, J. D.; BLACK, M. Seeds: physiology of development and germination. New York: Plenum Press, 1994. 445 p. https://doi.org/10.1007/978-1-4899-1002-8_9

CARVALHO, N.; NAKAGAWA, J. Sementes: ciência, tecnologia e produção. 4. ed. Jaboticabal: FUNEP, 2000. 588 p. https://doi.org/10.17801/0101-3122/rbs.v22n1p185-192

KOOLACHART, R.; JOGLOY, S.; VORASOOT, N.; WONGKAEW, S.; HOLBROOK, C. C.; JONGRUNGKLANG, N.; KESMALA, T.; PATANOTHAI, A. Rooting traits of peanut genotypes with different yield responses to terminal drought. Field Crops Research, v. 149, n. 1, p. 366-378, 2013. http://www.sciencedirect.com/science/article/pii/S0378429013002062 https://doi.org/10.1016/j.fcr.2013.05.024

KRATZ, D.; BASSACO, M. V. M.; NOGUEIRA, A. C.; Influence of water stress on germination of Zeyheria Montana. Journal of Biotechnology and Biodiversity, v. 4, n. 2, p. 140-145, 2013. https://sistemas.uft.edu.br/periodicos/index.php/JBB/article/view/416

LABOURIAU, L. G. A germinação das sementes. Washington: Secretaria Geral da Organização dos Estados Americanos, 1983. 174p. 
MAGUIRE, J. D. Speed of germination-aid in selection and evaluation for seedling emergence and vigor. Crop Science, v. 2, n. 1, p. 176-177, 1962.

https://dl.sciencesocieties.org/publications/cs/abstracts/2/2/CS0020020176

https://doi.org/10.2135/cropsci1962.0011183X000200020033x

MORADI, P.; FORD-LLOYD, B.; PRITCHARD, J. An evaluation of germination efficiency in a range of genotypes of Thymus species differing in susceptibility to drought. International Journal of Biosciences, v. 5, n. 10, p. 134-145, 2014. http://dx.doi.org/10.12692/ijb/5.10.134-145

PELEGRINI, L. L.; BORCIONI, E.; NOGUEIRA, A. C.; KOEHLER, H. S.; QUOIRIN, M. G. G. Efeito do estresse hídrico simulado com nacl, manitol e peg (6000) na germinação de sementes de Erythrina falcata Benth. Ciência Florestal, v. 23, n. 2, p. 513-521, 2013.

http://www.scielo.br/scielo.php?script=sci_arttext\&pid=S1980-50982013000200511\&lang=pt

https://doi.org/10.5902/198050989295

PEREIRA, J. W. L.; ALBUQUERQUE, M. B.; MELO FILHO, P. A.; NOGUEIRA, R. J. M. C.; DE LIMA, L. M.; SANTOS, R. C. Assessment of drought tolerance of peanut cultivars based on physiological and yield traits in a semiarid environment. Agricultural water management, v. 166, p. 70-76, 2016.

https://doi.org/10.1016/j.agwat.2015.12.010

PEREIRA, J. W. L.; MELO FILHO, P. A.; ALBUQUERQUE, M. B.; NOGUEIRA, R. J. M. C.; SANTOS, R. C. Mudanças bioquímicas em genótipos de amendoim submetidos a déficit hídrico moderado. Revista Ciência Agronômica, v. 43, n. 4, p. 766-773, 2012. http://www.scielo.br/scielo.php?script=sci_arttext\&pid=S180666902012000400019\&lang=pt https://doi.org/10.1590/S1806-66902012000400019

ROSA, L. S.; FELIPPI, M.; NOGUEIRA, A. C.; GROSSI, F. Avaliação da germinação sob diferentes potenciais osmóticos e caracterização morfológica da semente e plântula de Ateleia glazioviana baill (timbó). Revista Cerne, v. 11, n. 3, p. 306-314, 2005. http://www.redalyc.org/pdf/744/74411309.pdf

SANTOS, R. C.; FREIRE, R. M. M.; LIMA, L. M. O Agronegócio do Amendoim no Brasil. 2. ed. rev. e ampl. - Brasília, DF : Embrapa, 2013, 585P.

STEINBRECHER, T.; LEUBNER-METZGER, G. Tissue and cellular mechanics of seeds. Current opinion in genetics \& development, v. 51, p. 1-10, 2018. https://doi.org/10.1016/j.gde.2018.03.001

VILLELA, F. A.; DONI FILHO, L.; SIQUEIRA, E. L. Tabela do potencial osmótico em função da concentração de polietileno glicol 6000 e da temperatura. Pesquisa Agropecuária Brasileira, v. 26, n. 11-12, p. 1957-1968, 1991. https://seer.sct.embrapa.br/index.php/pab/article/view/3549

WANG, W. Q.; MØLLER, I. M.; SONG, S. Q. Proteomic analysis of embryonic axis of Pisum sativum seeds during germination and identification of proteins associated with loss of desiccation tolerance. Journal of proteomics, v. 77, p. 68-86, 2012. https://doi.org/10.1016/j.jprot.2012.07.005

ZHANG, B.; SU, L.; HU, B.; LI, L. Expression of AhDREB1, an AP2/ERF Transcription Factor Gene from Peanut, Is Affected by Histone Acetylation and Increases Abscisic Acid Sensitivity and Tolerance to Osmotic Stress in Arabidopsis. International journal of molecular sciences, v. 19, n. 5, p. 1441, 2018. http://dx.doi.org/10.3390/ijms19051441 